\title{
SIP-Based Vertical Handover Scheme with Bicasting
}

\author{
Toktam Hemmati*, Mohammad Hossein Yaghmaee Moghadam** \\ * Department of Computer Engineering, Islamic Azad University of Mashhad (IAUM), Iran \\ **Department of Computer Engineering of Ferdowsi University (FUM), Iran \\ toktam.hemmati@gmail.com,yaghmaee@ieee.org
}

\begin{abstract}
Due the complexity of the wireless environments, single technology cannot be efficient to provide demands of mobile users. Hence, in cooperation with heterogeneous wireless technologies, the next generation of wireless systems makes it possible to communicate everywhere. Providing high performance and data rate is what this cooperation has promised to meet. So issues related to the heterogeneity of wireless environment like handover should be addressed. With the appearance of overlapping wireless networks, MNs have to switch their connections between different access technologies having different capabilities and characteristics. In this case, the handover process is more complex and is known as Vertical Handover. In this paper, we focus on the vertical handover management and use an extension of SIP to support vertical handover with bicasting. Delay reduction and SIP signaling messages overhead reduction are the challenges we have tried to improve with our proposed method. To evaluate our proposed method, we use the OPNET Modeler 14.5 and it's Performance for real-time service besides examining the VoIP. Simulation has been performed on UMTS/WLAN networks. The results show that our technique can improve latency and throughput.
\end{abstract}

Keywords - Mobility Management, Vertical Handover, Session Initiation Protocol (SIP), Heterogeneous Wireless Network, Realtime Applications

\section{INTRODUCTION and RELATED WORK}

Increasing demand for high speed data access, also equipping users with advanced and smart device with multimedia capabilities and multiple radio interfaces have resulted in widespread deployment of heterogeneous wireless networks domains. This enables the users to connect to different network types such as WIMAX, WLAN, UMTS, etc. in order to choose it regarding cost and quality of service and other priorities. Due to the different access technologies and protocols, various network architectures and application services, integration of heterogeneous networks often presents some considerable challenges including mobility management demand for seamless handover, continuity of data traffic and multimedia sessions.

Mobility management contains two components: location management and handover management. Location management enables the system to track the locations of mobile nodes (MNs) between consecutive communications. Handover, also known as Handoff, is an event taking place whenever a MN moves from one wireless cell to another, abandoning the connection with the first base station and getting attached to the second one. When a handover occurs within the domain of a single wireless access technology, the process is known as horizontal handover. In contrast, vertical handover is a term referring to the handover among heterogeneous wireless access network technologies [4]. Figure 1 illustrates both horizontal and vertical handover events.

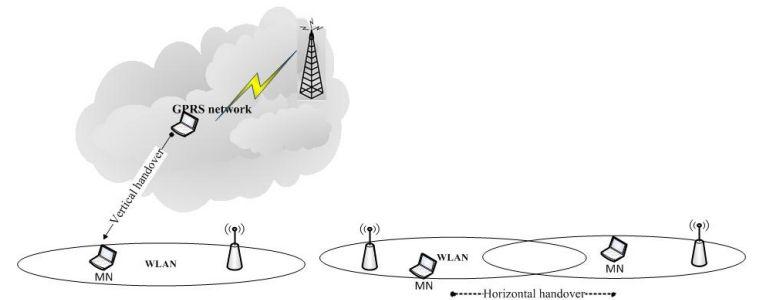

Figure 1. The horizontal and vertical handover

The integration of different wireless network technologies is needed to provide a "seamless" interoperability; integration and convergence among these heterogeneous technologies and, therefore, the use of vertical handover (VHO) techniques are required. VHO process is divided into three parts: (i) Handover information gathering, (ii) Handover decision, and (iii) Handover execution [1], [4].

The handover information gathering phase collects network information and also the information about the rest of the components of the system such as network properties, mobile devices, access points, and user preferences. This phase receives different names such as handover information gathering, system discovery, system detection. In this phase, the information is collected to be used and processed for making decisions in the handover decision phase. The handover decision phase is one of the most critical processes during the handover. This phase is also known as network selection or handover preparation. This phase is responsible for evaluating and deciding the most appropriate network in order to fulfil both the user and the system needs and suitable communication networks. The final phase performs the handover itself; moreover this phase should also guarantee a smooth session transition process. After the collection of data in the first phase and choosing the candidate network by the second phase, implementation phase will establish a connection to the new network.

When performing vertical handovers, the mobility management protocol must not only provide location transparency, but also network transparency. To obtain session continuity, there are two main approaches. Either to solve it 
on the network layer with Mobile IP or on the application layer with augmented existing protocols such as H.323 or SIP. Both network and application layer solutions have their advantages and disadvantages, but due to the flexibility and convenience of use, application layer implementations are considered.

SIP has been used as a signaling protocol for multimedia session management in the Internet, it has also been considered as an alternative mobility management protocol at the application layer for heterogeneous networks. Thus, SIP based mobility management could potentially use an available operational infrastructure, which would facilitate its fast deployment. It is desired that data sessions maintained by the mobile user stay alive and real-time sessions still have acceptable quality and moreover the handover spans for the shortest possible time. Since SIP has got text-based nature; message size in SIP is a challenge to keep signaling delay low while using SIP protocol as a management protocol. So, several studies have been done for analyzing the call setup latency in wireless networks.

References [12] and [14] are introduced as a Back-to-Back user agent (B2BUA) or a Session Border Controller (SBC) which acts as an RTP proxy to forward the media packets between $\mathrm{MN}$ and $\mathrm{CN}$. Making standard handover procedure depending on operator entities such as B2BUAs and SBCs, restricts the possible use of SIP and SIP mobility in nonoperator environments. Acting B2BUA/SBC as an RTP proxy can become a vulnerable hot spot. In addition, bridging the calls can become a bottleneck in terms of scalability.

The idea of [16] differs from the previous schemes because of being able to provide handover functionality without the need for new entities in the access networks or subdomains. It can either be used directly between two end nodes, or just by using an intermediate node such as a B2BUA. If the $\mathrm{CN}$ also supports the Handover Extension, media handover can be performed directly from the $\mathrm{CN}$ and not by the B2BUA. Thus, in a network where one can assume Handover Extension is supported by all the nodes, it will be possible to omit the use of a B2BUA as an anchor point.

Achour et al. [2] combine a multihoming protocol (SHIM6), which ensures a seamless network change, with the IMS architecture, allowing the establishment of multimedia sessions with the quality of service. Authors implement a new proxy SIP (P-SIP) inside the terminal to manage the signaling procedures. The proposed scheme can manage the interdomain mobility of a terminal in two modes: reactive and proactive. The reactive mode reacts to an event once it has already happened; the proactive mode predicts that an event has a strong chance of happening and acts, accordingly. Despite the advantages of this method, it suffers from packet loss.

Reaz at el. [9] propose an end-to-end mobility management scheme, Multi-class SIGMA (mSIGMA), that performs soft handover for inter-class and intra-class mobility in wireless networks. Our analysis shows mSIGMA performs seamless handover across the networks with low delay and packet loss.
In this paper, we propose a seamless vertical handover with a method based on session initiation protocol (SIP) using bicasting. This process exploits the link-layer triggers and the new SIP "handover" header to reduce handover delay and packet loss and makes use of this characteristic in which most of the fields in INVITE messages as well as re-INVITE messages are duplicated when a set of SIP messages are exchanged during session re-setup procedure in order to reduce the delay in transmitting SIP message.

The paper is outlined as follows; in the next section we will discuss our proposed method in detail. Section III presents the simulation model and its results. Finally, section IV concludes the paper.

\section{The PROPOSED METHOD}

The subject of mobility in SIP has already been studied for some time. The first solution was suggested by Wedlund and Schulzrinne in 1999 [19]. When the MN moves from one network to another, it simply sends another INVITE (called re-INVITE) to its $\mathrm{CN}$ to tell it about its new IP address. When a new INVITE message is sent to change an existing dialog, not only a description of the changes but also a full description of the session is sent. Major challenges using re-INVITE are the handover delay and SIP re-signaling overhead and SIP message size considering its text-based nature. So when this protocol is used as a management protocol, delay reduction and SIP signaling overhead messages reduction, are the challenges that we have tried to improve by using a new form of SIP header fields and optional field in SIP headers as a "make before break" way. We also use bi-casting to reduce loss and latency during handover. As you know, most of the fields in the INVITE message and the re-INVITE message are duplicated and one of the reasons for the delay in establishing a session SIP (re-setup) is the size of the SIP text-based messages. So, we replace the re-INVITE message with the compared message (CP) containing essential information like new call-ID and IP address and other header fields and new fields to reduce the transmission delay of SIP messages.

The new SIP 'handover' header is defined, as follows: REQURE: HANDOVER: add|del; IP= ip_address

This new header will be inserted into SIP re-INVITE method. This header instructs $\mathrm{CN}$ to add or delete the IP address indicated (ip_address) to or from the associated tables used for SIP signaling and media streams. Indeed, the 'add' flag will inform $\mathrm{CN}$ to start bicasting to $\mathrm{MN}$, in which $\mathrm{CN}$ will duplicate and transmits the identical media streams to $\mathrm{MN}$ and the 'del' flag instructs the $\mathrm{CN}$ to stop bicasting. For backward compatibility with the current SIP protocol, the re-INVITE message may include the 'require' header. If the $\mathrm{CN}$ cannot support the SIP handover with bicasting, it will respond to MN with "420 (Bad Extension)". In this case, MN may try to perform the exiting SIP handover.

During handover, bi-casting media streams to both access networks of the session's device may be beneficial to ensure a seamless session handover. In addition, the bi-casting media streams can also improve the quality of the services of realtime services according to the new access network 
characteristics. Bi-cast can be defined as the separation of a packet stream destined to a mobile node into two streams. If the mobile node connects two systems at the same time, a mobile node receives two streams simultaneously. Bi-cast is effective in reducing handover delay and packet loss rate. The start and end of bicasting of our proposed method is illustrated in Figure 2.

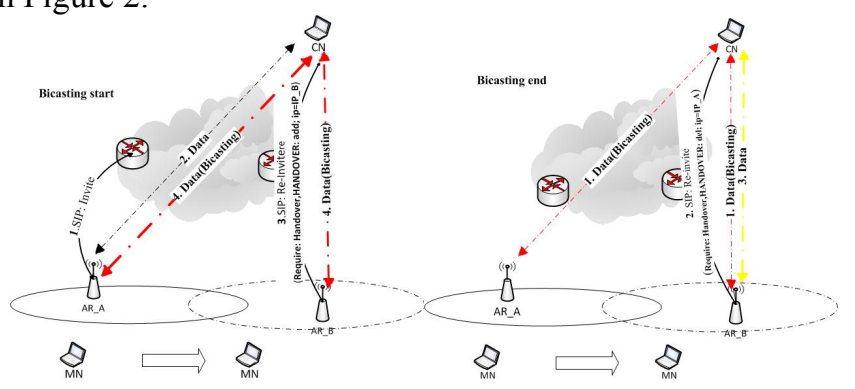

Figure 2. Starting and ending of bicasting

For the bi-casting action, it is necessary for the media streams bi-casting to be comprehensible for the methods based on SIP, and CN should also have the bicasting capabilities. We assumed that an $\mathrm{MN}$ is exploited by the link-layer triggers such as Link-Up and Link-Down. When the MN enters the handover region, it will initiate the handover operations with the help of link-layer triggers (sending re-INVITE message with new header). Since the SIP cannot support the soft handover with bicasting, it should be noted that the existing SIP handover does not use link-layer information. The proposed procedures are illustrated in Figure 3.

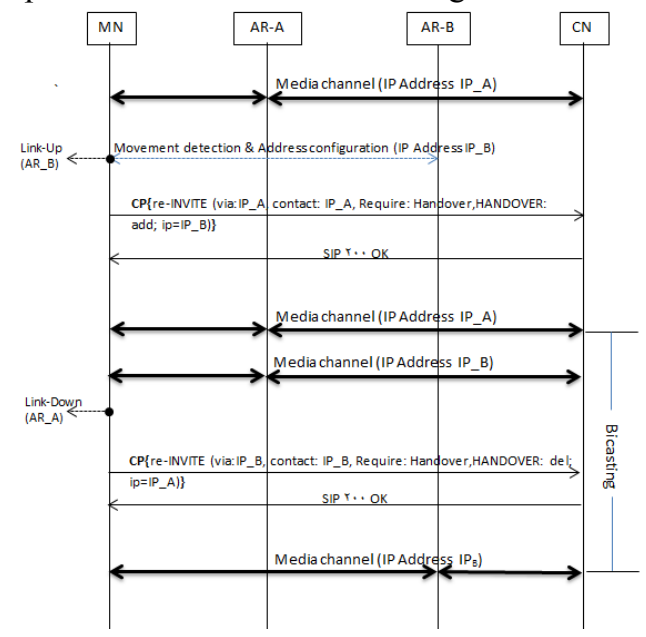

Figure 3. Data flow with Bicasting

As previously mentioned, most of the fields in header of INVITE message and the re-INVITE message are equivalent except for some number of fields such as the new IP address and the contact header.

In order to reduce the delay in transmitting SIP message between the $\mathrm{MN}$ in the new network and the $\mathrm{CN}$, in all of the steps mentioned above, MN may only extract the inconsistent fields between the header in old and new INVITE messages and send the inconsistent fields to the $\mathrm{CN}$, instead of sending the complete re-INVITE message directly [13]. Finally the host agent on the MN re-establishes the SIP session between the $\mathrm{MN}$ and $\mathrm{CN}$. This method requires the existence of host agent on both $\mathrm{MN}$ and $\mathrm{CN}$. MN uses it to extract different headers (CP) and sends it to and on $\mathrm{CN}$ in order to restore the whole re-INVITE message. When $\mathrm{MN}$ initiates a SIP session, a host agent on the $\mathrm{MN}$ and $\mathrm{CN}$, keeps the whole INVITE message.

\section{III.SIMULATION and EVALUATION RESULTS}

In this section, we describe the simulation model and evaluate our proposed method. The simulation model is designed using OPNET Modeler 14.5. Fig. 4 depicts the model used for simulation. Simulation environment is the combination of two networks (UMTS and WLAN) connected to a core network. Core network is a network with IP multimedia subsystem (IMS) framework.

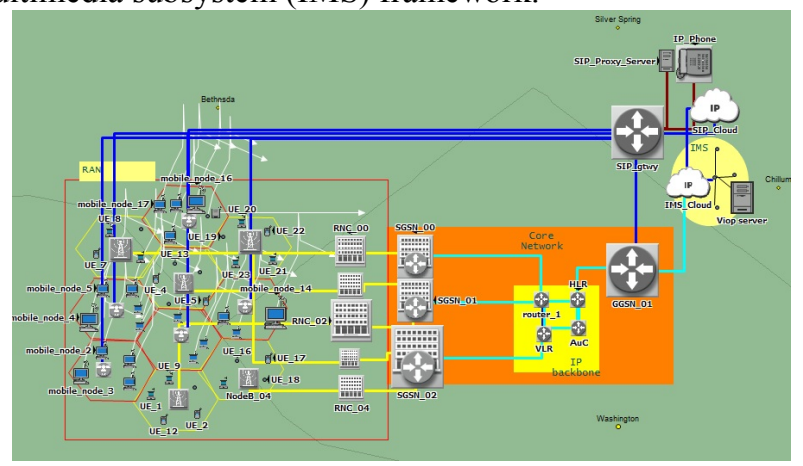

Figure 4. Simulation network design (UMTS/WLAN)

Simulation time is assumed to be $60 \mathrm{~min}$ for the VoIP application with G.711 or G.726 codec. The background traffic is implemented as file transfer and web browsing activities, mail services and....

To evaluate and analyse the performance of the proposed method, three different scenarios are simulated with the same architecture, but each one uses various handover policies. In the first scenario, a typical design is formed which uses existent SIP handover. The second scenario uses our proposed method with bicasting. Finally, the third scenario is a combination of our proposed method with bicasting and appropriate management of MAC layer in maintaining the physical layer resources. In fact, the physical layer uses OFDM. Therefore, the optimization of the physical layer characteristics can be effective in the optimization access network. Simulator compares the results of different scenarios. Figures 5 to 8 illustrate the improvement in various network parameters such as delay, packet loss, and jitter.

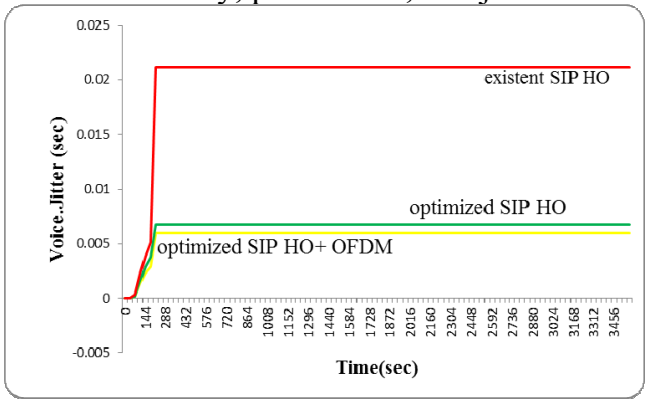

Figure 5. Compare the jitter in various scenarios 


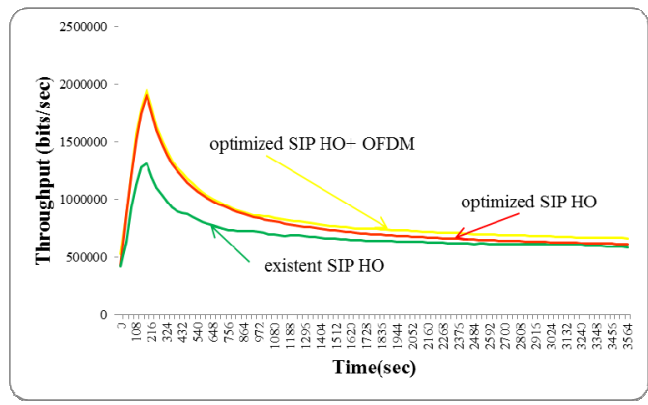

Figure 6. Comparison of network throughput in various scenarios
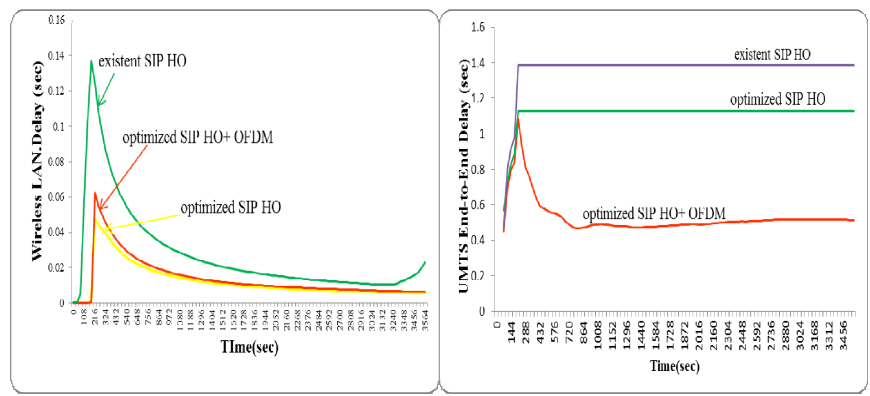

Figure 7. The delay improvement in UMTS \& WLAN

Increasing the throughput rate and reducing the delay of the proposed method shows that our proposed method is suitable for achieving the Quality of Service in vertical handover in the new generation of heterogeneous wireless networks.

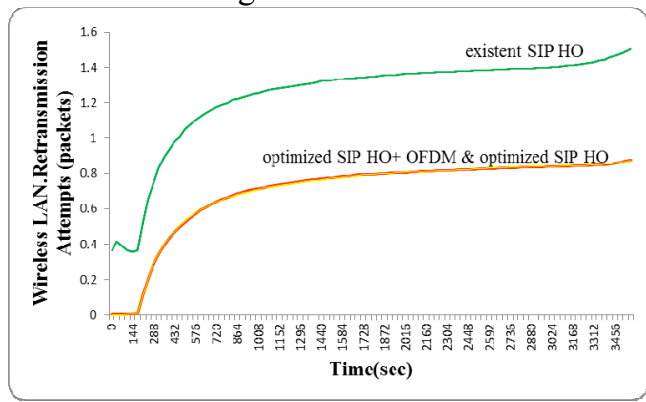

Figure 8. Retransmition attempt in WLAN

OPNET simulator can estimate the amount of MOS. It uses evaluating metrics such as Jitter. Figure 9 illustrates MOS in VoIP service.

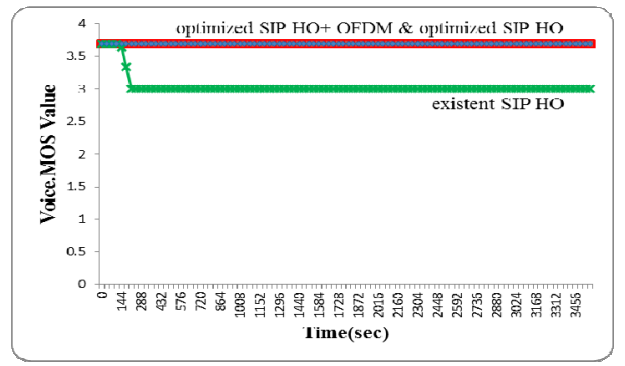

Figure 9. Comparison of the MOS Value of Voice over VoIP

\section{IV.CONCLUSIONS}

When users move between heterogeneous networks, Quality of their calls is affected by the handover delay. In this paper, we proposed a vertical handover method that uses SIP protocol and bicasting to improve SIP handover delay. Simulation results demonstrate that using this method can improve SIP handover. But, Since the start and end time of Bicasting highly influence limited resources of network such as network bandwidth backhaul and network buffers, providing a method to determine the most optimal time for beginning and ending the Bicasting could be an appropriate subject for future study. SIP Security issue can be interesting, too. In this method, SIP security techniques can be used to improve its security weaknesses.

\section{REFERENCES}

[1] M. Zekri, B. Jouaber, and D. Zeghlache, "A review on mobility management and vertical handover solutions over heterogeneous wireless networks," Elsevier, vol. 35, pp. 2055-2068, Oct. 2012.

[2] A. Achour, K. Haddadou, B. Kervella, and G. Pujollo, "A SIP-SHIM6based solution providing interdomain service continuity in IMS-based networks," IEEE Communications Magazine, vol. 50, pp. 109-119, July 2012.

[3] I. F.Akyidiz, J. Xie, and S. Mohanty, "A survey of mobility management in next-generation all-IP-based wireless systems, " IEEE Wireless Communications magazine, vol. 11, pp. 16-28, Aug. 2004.

[4] J. Marquez-Barja, C. T.Calafate, J.-C. Cano, and P. Manzonu, "An overview of vertical handover techniques: algorithms, protocols and tools," Elsevier, vol. 34, pp. 985-997, June 2011.

[5] W.Ma and Y.Fang, "Dynamic hierarchical mobility management strategy for mobile IP networks," IEEE Journal on Selected Areas in Communications, vol. 22, pp. 664-676, May 2004.

[6] H. Fathi and R. Prasad, "Mobility management for VoIP in 3G systems: evaluation of low-latency handoff schemes," IEEE Wireless Communications magazine, vol. 12, pp. 96-104, April 2005.

[7] M. Bernacchi, F. Cacace, G. Lannello, and M. Vellucci, "Mobility Management for VoIP on Heterogeneous Networks: Evaluation of Adaptive Schemes," Mobile Computing, IEEE Transactions on, vol. 6, pp. 1035-1047, Sept. 2007.

[8] T. T. Kwon, M. Gerla, S. Das, and S. Das, "Mobility management for VoIP service: Mobile IP vs. SIP," IEEE Wireless Communications magazine, vol. 9, pp. 66-75, Oct. 2002.

[9] A. Reaz, S. Ferdousi, and M. Atiquzzaman, "mSIGMA: An Efficient Handoff Scheme for Multiclass Networks," IEEE Systems Journal, vol. 4, pp. 210 - 220, June 2010.

[10] S. J. Koh and W. Hyum, "mSIP: Extension of SIP for Soft Handover with Bicasting," IEEE communications Letters, pp. 532 - 534, July 2008.

[11] S. Pack, G. Park, Y. Kim, and W. Lee, "Reducing Call Setup in mobile VoIP systems," IEEE communications Letters," vol. 15, Oct. 2011.

[12] E. S. Boysen and T. Maseng, "Seamless Handover in Heterogeneous Networks using SIP A Proactive Handover Scheme with the Handover Extension," International Journal on Advances in Internet, vol. 2, pp. 184-193, June 2009.

[13] O. A. El-Mohsen, H. A. M.Saleh, and S. Elramly, "SIP-Based Handoff Scheme in Next Generation Wireless Networks," in Proc. NGMAST, 2012, p. 131.

[14] S. Salsano and A. Polidoro, "SIP-based mobility management in next generation networks," IEEE Wireless Communications magazine, vol. 15, pp. 92-99, April 2008.

[15] M. Rahnema, UMTS NETWORK PLANNING, OPTIMIZATION, AND INTER-OPERATION WITH GSM, John Wiley \& Sons (Asia) Pte Ltd, 2008.

[16] E. S. Boysen and J. Flathagen, "Using SIP for seamless handover in heterogeneous networks," in Proc. ICUMT, 2011, P. 1.

[17] M. Stemm and R. H. Katz, "Vertical handoffs in wireless overlay networks," Springer mobile networks and applications, vol. 3, pp. 335350, 1998.

[18] K. Qiang and C. Yewen, "HBSIP: A SIP-based mobility management for seamless handover," in Proc. IC-BNMT, 2009, p. 729.

[19] E.Wedlund and H. Schulzrinne," Mobility support using SIP," in Proc WoWMoM'99, 1999, p. 76.

[20] rfc3261. Available: http://www.ietf.org/rfc/rfc3261.txt 